\title{
The Mediterranean-style diet for the prevention of cardiovascular diseases
}

\author{
Michel de Lorgeril* and Patricia Salen \\ Laboratoire Nutrition, Vieillissement et Maladies Cardiovasculaires (NVMCV), Université Joseph Fourier, \\ Grenoble, France
}

\begin{abstract}
Objectives: To discuss present knowledge about Mediterranean diet and cardiovascular diseases.

Design: Review of existing literature.

Setting and Results: Epidemiological studies as well as randomised dietary trials suggest that Mediterranean diet may be important in relation to the pathogenesis (and prevention) of CHD. For instance, a striking protective effect of an ALA-rich Mediterranean diet was reported in the Lyon Diet Heart Study with a 50 to $70 \%$ reduction of the risk of recurrence after 4 years of follow-up in CHD patients. According to our current knowledge, dietary ALA should represent about 0.6 to $1 \%$ of total daily energy or about $2 \mathrm{~g}$ per day in patients following a Mediterranean diet, whereas the average intake in linoleic acid should not exceed $7 \mathrm{~g}$ per day. Supplementation with very-long-chain omega- 3 fatty acids (about $1 \mathrm{~g}$ per day) in patients following a Mediterranean type of diet was shown to decrease the risk of cardiac death by $30 \%$ and of sudden cardiac death by $45 \%$ in the GISSI trial.

Conclusions: In the context of a diet rich in oleic acid, poor in saturated fats and low in omega- 6 fatty acids (a dietary pattern characterising the traditional Mediterranean diet), even small doses of omega-3 fatty acids (about $1 \mathrm{~g} \mathrm{EPA}+$ DHA the form of fish oil capsules or $2 \mathrm{~g} \alpha$-linolenic acid in canola oil and margarine) might be very protective. These data underline the importance of the accompanying diet in any dietary strategy using fatty acid complements.
\end{abstract}

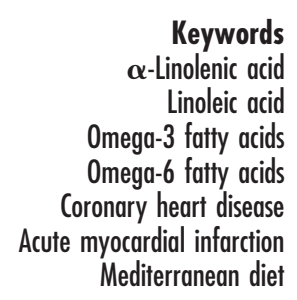

Keywords

-Linolenic acid Omega-3 fatty acids Omega-6 fatty acids Coronary heart disease Mediterranean diet
The concept of the Mediterranean diet originated from several observational studies in the 1950s, the main one being the Seven Countries Study initiated by Ancel Keys ${ }^{1}$. Taken as a whole, these studies showed that, despite a quite high fat intake (in variable amounts in the different Mediterranean countries; high in Greece, quite low in Tunisia, Morocco and in Spain and in Catalonia for instance), these populations had low to very low rates of coronary heart disease (CHD) (and other vascular diseases), of most types of cancer and of inflammatory and degenerative diseases, resulting in a long life expectancy. Because of the lack of striking differences in the traditional risk factors with other less protected populations (especially in terms of smoking, diabetes and high blood pressure), the Mediterranean dietary pattern was considered to be largely responsible for the good health observed in these regions ${ }^{2}$.

The main characteristics of the Mediterranean diet include an abundance of plant foods (vegetables, fresh and dried fruits, whole-grain cereals, nuts and legumes); olive oil as the principal source of fat; fish, egg, cheese and yoghurt, and poultry in low to moderate amounts; very low consumption of red meat, high-fat dairy products (saturated fats) and vegetable polyunsaturated (including trans fatty acids) oils and margarines; moderate consumption of wine ${ }^{2}$. However, despite these strong data, it remained that causal relationships could be demonstrated only by conducting clinical trials. We now have results of randomised trials supporting the theory that the Mediterranean diet is a very healthy diet.

\section{The Lyon Diet Heart Study}

The Lyon Diet Heart Study is a secondary prevention trial designed to test the hypothesis that a Mediterranean $\alpha$-linolenic acid (ALA) rich diet may improve the prognosis of patients having survived a first acute myocardial infarction $(\mathrm{AMI})^{3-6}$. The design, methods and results of the trial have been reported ${ }^{3-5}$. A striking protective effect of that Mediterranean diet was reported with a 50 to $70 \%$ reduction of the risk of recurrence after four years of followup $^{3-6}$. Briefly, as regards lipids, the experimental Mediterranean diet tested in the trial supplied less than $30 \%$ of energy from fats and less than $8 \%$ of energy from 
saturated fats. Regarding essential fatty acids, the intake of linoleic acid (LA), the main omega- 6 fatty acid, was restricted to $4 \%$ of energy and the intake of ALA, the main omega- 3 fatty acid, made up more than $0.6 \%$ of energy. In practical terms, the dietary instructions were detailed and customised to each patient ${ }^{3-6}$ and can be summarised as: more bread, more cereals, more legumes and beans, more fresh vegetables and fruits, more fish, less meat (beef, lamb, pork) and delicatessen, which were to be replaced by poultry; no more butter and cream, to be replaced by an experimental canola oil-based margarine. This margarine was chemically comparable with olive oil but slightly enriched in LA and mostly in ALA, the two essential fatty acids. Finally, the oils recommended for salad and food preparation were exclusively olive and canola (erucid acidfree rapeseed oil) oils. The scientific rationale for that 'dietary fat strategy' has been discussed elsewhere ${ }^{3-6}$. Briefly, it was hypothesised that, because the lowest rates of cardiovascular diseases in the world were observed in populations either following a Mediterranean diet or a diet low in $n-6$ fatty acids but rich in $n-3$ fatty acids, the best strategy to reduce the rate of complications in patients with established CHD should be to adopt an $n-3$ fatty acid-rich Mediterranean diet. Two other major components of the traditional Mediterranean diet, in addition to a low $n-6 /$ $n-3$ fatty acid ratio, are low saturated fat intake and high oleic acid intake. Patients also had to meet these two major criteria of a healthy diet. Thus, to meet the criteria of a Mediterranean diet, patients had to drastically reduce the consumption of foods rich in saturated (essentially animal) fat. Among vegetable oils, only olive oil (despite its lack of ALA) and canola oil (despite its moderate amounts of LA) have a fatty acid composition in line with our strategy. Thus, the patients were advised to use both oils. Because of their high content in LA, soybean, sunflower and walnut oils should not be used daily for food preparation and salad dressing. Peanut oil is too rich in saturated fatty acids and LA and linseed oil is too rich in polyunsaturated fatty acids. In theory, the best option could be to vary the use of several oils. However, when considering the difficult conditions of everyday life for many of our patients and their families, we decided to try and simplify our advice and to recommend the exclusive use of olive and canola oils.

This strategy was quite well accepted by the French patients who, at the end of the trial, were actually following a diet whose characteristics were close to that we envisaged, in theory, as the golden standard cardioprotective diet, at least in terms of lipid nutrients ${ }^{3-6}$.

This exclusive use of olive and canola oils (and of canola-oil based margarine instead of butter to spread on the bread) to prepare meals and salad was a major issue in that trial as it resulted in significant differences in the fatty acid composition of both circulating plasma lipids (essentially lipoproteins) and cell membrane phospholipids $^{3,7}$. The main differences between groups in platelet phospholipid fatty acids were not seen at the level of individual fatty acids (ALA is almost undetectable in cell membranes) but for the entire family of each group. Significant differences were also seen for the ratio of omega-6 ( $n-6)$ to omega-3 $(n-3)$ fatty acids ${ }^{3,7}$. When comparing the dietary fatty acids in the two groups, control patients did consume about $0.7 \mathrm{~g}$ of ALA per day against about $1.8 \mathrm{~g}$ in the experimental group, i.e. giving an LA/ALA ratio of about 10:1 in controls against about $4: 1$ in the experimental group. It is noteworthy that if the risk of recurrence in the experimental group was lower than in the control group, the risk in the control group was not high compared with previous studies indicating that the 10:1 ratio was, in theory, not so bad. Because the Mediterranean diet tested in that trial was different from the control diet in many other aspects than the LA/ ALA ratio (less saturated fat, more antioxidants from various sources, and probably more vitamins of the $\mathrm{B}$ group including folic acid, more vegetable proteins, and so on), the next question was to try and specify the exact role of ALA in the cardioprotection observed in the trial. Using multivariate analyses and adjustment for several confounders, we found that the plasma ALA levels measured two months after randomisation were significantly (and inversely) associated with the risk of recurrence, and in particular of fatal recurrence ${ }^{6}$. It could be said, however, that it is not ALA per se that was protective but the very-long-chain $n-3$ fatty acids derived from ALA, eicosapentaenoic and docosahexaenoic acids (DHA), which were also increased in the plasma of experimental patients ${ }^{3,7}$. These very-longchain $n-3$ fatty acids have indeed been demonstrated to prevent ventricular fibrillation (VF) and sudden cardiac death (SCD) in animal experiments ${ }^{8,9}$ and in human trials ${ }^{10,11}$. However, in the Lyon trial, these fatty acids were not significantly (borderline non-significant with DHA) associated with a lower risk, which suggests that ALA was the main protective factor. Also, a specific antiarrhythmic effect of ALA itself was reported in the animal studies where it was tested ${ }^{9}$. Finally, dietary ALA was shown to be inversely related to the risk of SCD among the 76763 women participating in the Nurses' Health Study ${ }^{12}$ suggesting that ALA itself could have antiarrhythmic properties. It does not mean, however, that the benefits of ALA are not due, at least partly, to its conversion into very-long-chain $n-3$ fatty acids, and further studies are required to differentiate the individual effects of each $n-3$ fatty acid in the specific context of myocardial ischaemia and ventricular arrhythmias.

In fact, the potential effects of ALA on cardiovascular diseases were discovered when it was reported that populations with a high proportion of ALA in plasma lipids (the Greek and Japanese cohorts of the Seven Countries Study, for instance) are apparently protected from cardiovascular diseases ${ }^{1,13}$, and when it was found that these low-risk populations consume foods rich in ALA ${ }^{14,15}$. Another important point to encourage the consumption of 
ALA and to reduce the intake of LA (with a significant decrease in the omega-6/omega-3 ratios) came from the observation that the lowest ex vivo platelet aggregation measured in humans in response to adenosine diphosphate, a possible indicator of the risk of acute CHD events, was recorded with a dietary LA/ALA ratio of about $4: 1^{16,17}$, i.e. much lower than that of the present Western diet and also lower than what many experts recommend at present. This pointed out the potential importance of ALA to prevent the thrombotic complications of $\mathrm{CHD}^{18}$.

Other works have suggested that eating nuts (a major source of ALA among the Mediterranean especially the Greek) was associated with a diminished risk of $\mathrm{CHD}^{19}$. Potentially protective constituents of nuts include ALA, folates, magnesium, potassium, fibre, vitamin E, arginine and favourable lysine-to-arginine and methionine-toarginine ratios ${ }^{19}$. One noteworthy point is that the fatty acid composition of the lipids in walnuts (also called English walnut or noix de Grenoble or Californian nut) is apparently the same on both sides of the Atlantic, as the concentrations of ALA, LA and oleic acid measured in our laboratory are respectively $13.4,60.5$ and $15.6 \%$ in the noix de Grenoble and 12.9, 62.5 and 13\% in the Californian nut.

It is clear that beside their fatty acid composition, certain nuts may be important because they provide large amounts of arginine (even more than meat) with the major advantage that they are quite poor in methionine (the precursor of the vasculo-toxic homocysteine) as compared with meat and fish. This is especially true for walnut, almond and hazelnut, which are habitually eaten in quite great amounts by Mediterranean populations. The last, but certainly not least, point is the relatively high content in folates of most nuts. Low serum folate levels on one hand and high homocysteine levels hand on the other ${ }^{20,21}$ have been clearly associated with an increased risk of CHD. The results of recent trials were, however, not consistent with a decreased risk of CHD following homocysteine-lowering treatment. This suggests that prevention of cardiovascular diseases (as well as of cognitive decline) by (a mix of) vitamins B should probably be started very early in life to be effective and very probably in association with a global protective diet rather than in the form of capsules. Thus, although there is no room here to fully discuss each of these points, nuts (which are both rich in ALA and important in the Mediterranean diet) can obviously be included as part of a healthy diet. In contrast, and because walnut oil is too rich in LA and does not contain the other healthy nutrients present in the fruit nut itself, daily consumption of that oil is not advisable. There are different ways to obtain $2 \mathrm{~g}$ of ALA per day in the diet, as in the Lyon Diet Heart Study, without using ALA-containing capsules or fortified foods. The simplest (and easiest) way is to use canola oil for food preparation and salad dressing. Since $100 \mathrm{~g}$ of canola oil provide about $8 \mathrm{~g}$ of ALA, two small (US) tablespoons provide about $2 \mathrm{~g}$ of ALA. The canola-oil based margarine, which contains about $5 \mathrm{~g}$ of ALA per $100 \mathrm{~g}$ of margarine, may be useful. Obtaining $2 \mathrm{~g}$ of ALA requires $35 \mathrm{~g}$ of margarine, i.e. about six teaspoons. Alternatively, one can have one tablespoon of canola oil (with salad, for instance) and 2 teaspoons of canola margarine with a piece of bread. Both canola oil and margarine can be used in association with olive oil (that does not contain ALA) for food preparation because of the large amounts of oleic acid and flavonoids it contains, and also because of its particular taste. Regarding nuts, to obtain $2 \mathrm{~g}$ of ALA, about 6 medium size English walnuts (or noix de Grenoble) are needed. If one likes English walnuts enough to eat them every day (as Mediterranean people used to do), it is possible to use exclusively olive oil for food preparation. A good (and definitely tasty) way is to add the nuts to salad. One can also use ground linseeds (not linseed oil) with salad or other green leafy vegetables, knowing that $100 \mathrm{~g}$ of ground linseeds provide about $23 \mathrm{~g}$ of ALA. Thus, only one tablespoon of ground linseeds provides about $2 \mathrm{~g}$ of ALA.

It has been the merit of Simopoulos and colleagues to show that many green leafy vegetables (such as purslane) largely consumed around the Mediterranean basin are a major source of ALA for the Cretan population at that period ${ }^{14}$. The Cretan diet is also rich in antioxidants. This is a major point since, because of its three double bonds, ALA is highly sensitive to oxidation and a high intake of ALA must be combined with a high intake of antioxidants to protect it from oxidation. The same authors also reported that eggs from range-fed Greek hens (which make a feast of purslane and other ALA-rich fresh green grass) are richer in ALA and other $n-3$ fatty acids than eggs bought in US supermarkets which are, in turn, usually rich in $\mathrm{LA}^{15}$. The large difference in the fatty acid composition of the two types of eggs was indeed also due to the fatty acid composition of the industrial feedstuff given to US hens. This suggested that egg yolk might have been a considerable source of ALA and other $n-3$ fatty acids for people living in the Mediterranean area. The point is important because this population usually does not consume large amounts of marine products rich in verylong-chain $n-3$ fatty acids. Eating purslane (or other equivalent ALA-rich leafy vegetables) to obtain large amounts of ALA is not very easy, in particular because purslane (or its equivalents) is not available in many areas. In addition, one big portion ( $100 \mathrm{~g}$ of purslane) provides less than $0.4 \mathrm{~g}$ of ALA. Thus, for most adults, purslane should be associated with either walnuts or a salad dressing containing canola oil. One advantage of eating green leafy vegetables (and not only purslane) is to increase the diversity of the diet, which is probably a major component of any healthy diet, and one advantage of eating eggs from hens fed with ALA-rich grains is to increase the intake of DHA, the longest omega-3 fatty acid otherwise only found in fatty fish and fish oil. 


\section{Fish oil and Mediterranean diet}

The theory that eating fish, a major component of the traditional diet of several Mediterranean populations (in South Italy, Sicily, Greece, Catalonia and also Portugal, for instance) may protect against cardiac death is derived from the results of a secondary prevention trial, the Diet And Reinfarction Trial (DART), which showed a significant reduction in total and cardiovascular (CV) mortality (both by about 30\%) in patients who consumed at least two servings of fatty fish per week ${ }^{10}$. The authors suggested that the protective effect of fish might be result from a preventive effect on VF, since no benefit was observed on the incidence of non-fatal AMI. The hypothesis was consistent with experimental evidence suggesting that the very-long-chain $n-3$ fatty acids, the dominant fatty acids in fish oil and fatty fish, have an important effect on the occurrence of VF in the setting of myocardial ischaemia and reperfusion in various animal models, both in vivo and in vitro ${ }^{8}$. Recently, Billman and colleagues, using an elegant in vivo model of SCD in dogs, demonstrated a striking reduction of $\mathrm{VF}$ after intravenous administration of pure $n-3$ fatty acids, including the very-long-chain fatty acids present in fish oil and ALA, their parent $n-3$ fatty acid occurring in some vegetable oils ${ }^{9}$. Another important aspect of the involvement of $n-3$ fatty acids in SCD concerns their role in the metabolism of eicosanoids. In competition with $n-6$ fatty acids, they are the precursors to a broad array of structurally diverse and potent bioactive lipids (including eicosanoids, prostaglandins and thromboxanes), which are thought to play a role in the occurrence of VF during myocardial ischaemia and reperfusion $^{22,23}$.

Other interesting clinical data show suppression (by more than 70\%) of ventricular premature complexes in middle-aged patients with frequent ventricular extrasystoles randomly assigned to take either fish oil or placebo $^{24}$. Also, survivors of $\mathrm{AMI}^{25}$ and healthy men ${ }^{26}$ receiving fish oil were shown to improve their measurements of heart rate variability, which suggests that there are other mechanisms by which $n-3$ fatty acids may be antiarrhythmic. In fact, parasympathetic cardiac tone is thought to provide protection against $\mathrm{VF}^{27}$.

Support for the hypothesis of a clinically significant antiarrhythmic effect of $n-3$ fatty acids in secondary prevention of $\mathrm{CHD}$, as put forward in $\mathrm{DART}^{10}$, came from epidemiological studies ${ }^{28}$. In particular, in a large prospective study (more than 20000 participants with a follow-up of 11 years), Albert et al. examined whether fish might have antiarrhythmic properties and prevent $\mathrm{SCD}^{29}$. They found that the risk of SCD was 50\% lower for men who consumed fish at least once a week than for those who had fish less than once a month. Interestingly, the consumption of fish was not related to non-sudden cardiac death, which suggests that the main protective effect of fish (or very-long-chain $n-3$ fatty acids) is related to an effect on arrhythmia. Finally, in a recent trial conducted in patients with implantable cardiac defibrillator, Leaf reported a $40 \%$ reduction of recurrent ventricular arrhythmias following fish oil capsules in a double blinded trial $^{30}$. It is noteworthy, however, that in another recent publication reporting fish oil supplementation in the same type of patients, the results were negative ${ }^{31}$. Beside major technical limitations (too small sample size resulting in randomised groups not well balanced for some baseline risk factors and under-powered statistical analyses) especially in one trial with only 200 patients $^{31}$, there is no clear explanation for that discrepancy between trials. Obviously, some major dietary confounders (background marine and plant omega- 3 fatty acid intake, omega- 6 fatty acid intake, trans fatty acid intake, saturated fatty acid intake), which could greatly interfere with the omega-3 fatty acids given with the capsules, were curiously not measured in these trials and not included (for minimal adjustments) in the calculations of the risks.

The GISSI-Prevenzione trial was aimed at helping in addressing the question of the health benefits of foods rich in very-long-chain $n-3$ fatty acids (and also in vitamin $\mathrm{E}$ ) and their pharmacological substitutes ${ }^{11}$. Patients ( $n=11324)$ surviving a recent AMI ( $<3$ months) were randomly assigned supplements of $n-3$ fatty acids ( $1 \mathrm{~g}$ daily), vitamin E (300 mg daily), both or none (control) for 3.5 years. The primary efficacy endpoint was the combination of death and non-fatal AMI and stroke. Secondary analyses included overall mortality, CV mortality and SCD. Treatment with $n-3$ fatty acids significantly lowered the risk of the primary endpoint (the relative risk decreased by 15\%). Secondary analyses provided a clearer profile of the clinical effects of $n-3$ fatty acids. Overall mortality was reduced by $20 \%$ and CV mortality by $30 \%$. However, it was the effect on SCD ( $45 \%$ lower) that accounted for most of the benefits seen in the primary combined endpoint and both overall and CV mortality. There was no difference across the treatment groups for non-fatal CV events, a result comparable to that of DART ${ }^{10}$.

A major point is that in that trial conducted in Italy, all patients were advised (before randomisation) to follow a Mediterranean type of diet after their AMI. In their report, the GISSI investigators confirmed that the patients of both groups actually did so; for instance, more than $80 \%$ reported that they consumed olive oil every day ${ }^{11}$. Thus we can say that in GISSI, the prevention of SCD resulting from the consumption of $1 \mathrm{~g}$ very-long-chain $n-3$ fatty acids was observed in patients following a Mediterranean diet as background diet. Whether they would have been protected in the same way with a non-Mediterranean diet is an open question. Another way of seeing the question is to ask whether or not the GISSI patients were relatively deficient in very-long-chain $n-3$ fatty acids at baseline. If they were, we can understand that even a small dose of $n-3$ fatty acids was so effective. In fact, recent 
(unpublished) data from the European IMMIDIET Project suggest that the Italian population could be relatively deficient in $n-3$ fatty acids as compared with British (South of London) and Belgian (Flemish) populations, thus confirming the hypothesis that a low dose of $n-3$ fatty acids was so effective in GISSI because the tested population was probably relatively deficient in $n-3$ fatty acids. Thus, in the context of a Mediterranean diet rich in oleic acid and poor in saturated and $n-6$ fatty acids, even a small dose of $n-3$ fatty acids (in the form of capsules) might be very protective. It is not sure whether the results would have been similar in another context, for instance in populations with high intake in $n-6$ and trans fatty acids. Finally, in a separate analysis, the investigators of GISSI analysed in their cohort (pooling together the randomised groups) the effect of adhering closely or not to the main Mediterranean diet principles ${ }^{32}$. They found that compared with patients in the worst Mediterranean diet score, the risk of dying (from any cause) for those in the best score was reduced by $50 \%$ after adjustment for age, sex, smoking, concomitant drug therapy and randomised treatment $^{32}$, a result very similar to that observed in the Lyon Diet Heart Study ${ }^{3,6}$.

\section{Conclusions}

A recent epidemiological study from Greece confirmed that following a Mediterranean diet considerably reduces the risk of premature death ${ }^{33}$. Indeed, Trichopoulou and co-workers reported, using a food-frequency questionnaire and a Mediterranean diet scale (higher score indicating a greater adherence to the Mediterranean diet) in more than 22000 adults in Greece, a reduction in total, cardiac and cancer mortality with a high degree of adherence to the Mediterranean diet ${ }^{33}$. The data are in line with previous observational ${ }^{1}$ and trial data ${ }^{3,6}$. As a matter of fact, several national and international expert committees, notably in Europe and $\mathrm{USA}^{34}$, have decided to definitely adopt the Mediterranean diet as the reference diet for the prevention of cardiovascular diseases.

The next very important question will be to precisely understand how that dietary pattern protects against CHD, and also most cancers and inflammatory diseases. We already have some answers with the biological (and well described cardioprotective) effects of omega-3 fatty acids, natural antioxidants, ethanol (and polyphenols), oleic acid and folic acid, all being basic nutrients of the Mediterranean diet. In addition, recent data indicate that this type of diet, taken as a whole rather than as an addition of individual protective nutrients, attenuates inflammation and coagulation process ${ }^{35}$, and that among patients with the metabolic syndrome, defined according to the NCEP ATP III criteria ${ }^{36}$, adoption of the Mediterranean diet was associated with a highly significant reduction of the risk of developing a metabolic syndrome and its associated risk of $\mathrm{CHD}^{37}$. Thus, even in the context of a medical condition typical of the modern world in both developed (at least one in five people had a metabolic syndrome in the USA in 2002) and developing countries, the Mediterranean diet pattern appears to be the prototype of a healthy diet. It should be emphasised to conclude that in most trials where the Mediterranean diet was advised to patients and their families, there were never major problems or difficulties to adopt the main principles of that dietary pattern. In addition, as seen in the Lyon Diet Heart Study, after the end of the trial, most patients continue to adhere to these principles. They usually do not come back to their previous dietary habits. This is a major point to support the view that actually dietary pattern is the ideal diet for people living in the Western industrialised world.

\section{References}

1 Keys A. Seven countries. A Multivariate Analysis of Death and Coronary Heart Disease. Cambridge, MA: Harvard University Press, 1980; 1-381.

2 de Lorgeril M. Mediterranean diet in the prevention of coronary heart disease. Nutrition 1998; 14: 55-7.

3 de Lorgeril M, Renaud S, Mamelle N. Mediterranean alphalinolenic acid-rich diet in secondary prevention of coronary heart disease. Lancet 1994; 343: 1454-9.

4 de Lorgeril M, Salen P, Martin JL. Effect of a Mediterranean type of diet on the rate of cardiovascular complications in coronary patients. Insights into the cardioprotective effect of certain nutriments. Journal of the American College of Cardiology 1996; 28: 1103-8.

5 de Lorgeril M, Salen P, Caillat-Vallet E. Control of bias in dietary trial to prevent coronary recurrences. The Lyon Diet Heart Study. European Journal of Clinical Nutrition 1997; 51: $116-22$.

6 de Lorgeril M, Salen P, Martin JL. Mediterranean diet, traditional risk factors and the rate of cardiovascular complications after myocardial infarction. Final report of the Lyon Diet Heart Study. Circulation 1999; 99: 779-85.

7 de Lorgeril M, Salen P. Modified Mediterranean diet in the prevention of coronary heart disease and cancer. World Review of Nutrition and Dietetics 2000; 87: 1-23.

8 McLennan PL, Abeywardena MY, Charnock JS. Reversal of arrhythmogenic effects of long term saturated fatty acid intake by dietary $n-3$ and $n-6$ polyunsaturated fatty acids. American Journal of Clinical Nutrition 1990; 51: 53-8.

9 Billman GE, Kang JX, Leaf A. Prevention of sudden cardiac death by dietary pure omega-3 polyunsaturated fatty acids in dogs. Circulation 1999; 99: 2452-7.

10 Burr ML, Fehily AM, Gilbert JF. Effects of changes in fat, fish, and fibre intakes on death and myocardial reinfarction: Diet And Reinfarction Trial (DART). Lancet 1989; 2: 757-61.

11 GISSI-Prevenzione Investigators. Dietary supplementation with $n-3$ polyunsaturated fatty acids and vitamin $\mathrm{E}$ after myocardial infarction: results of the GISSI-Prevenzione trial. Lancet 1999; 354: 447-55.

12 Albert C, Oh K, Whang W, Manson JE, Chae CU, Stampfer MJ, et al. Dietary alpha-linolenic acid intake and risk of sudden cardiac death and coronary heart disease. Circulation 2005; 112: $3232-8$.

13 Sandker GN, Kromhout D, Aravanis C. Serum cholesteryl ester fatty acids and their relation with serum lipids in elderly men in Crete and Netherlands. European Journal of Clinical Nutrition 1993; 47: 201-8.

14 Simopoulos AP, Norman HA, Gillapsy JE, Duke JA. Common purslane: a source of omega-3 fatty acids and antioxidants. 
Journal of the American College of Nutrition 1992; 11: 374-82.

15 Simopoulos AP, Salem N. $n-3$ fatty acids in eggs from rangefed Greek chickens. New England Journal of Medicine 1989; 321: 1412 .

16 Renaud S. Linoleic acid, platelet aggregation and myocardial infarction. Atherosclerosis 1990; 80: 255-6.

17 Renaud S, Nordoy A. Small is beautiful: alpha-linolenic and eicosapentanoic acids in man. Lancet 1983; 1: 1169.

18 Renaud S, de Lorgeril M. Dietary lipids and their relation to ischemic heart disease: from epidemiology to prevention. Journal of Internal Medicine 1989; 225: 39-46.

19 de Lorgeril M, Salen P, Laporte F. Potential use of nuts for the prevention and treatment of coronary heart disease: from natural to functional foods. Nutrition, Metabolism and Cardiovascular Diseases 2001; 11: 362-71.

20 Robinson K, Arheart K, Refsum H. Low circulating folate and vitamin B6 concentrations. Risk factors for stroke, peripheral vascular disease and coronary heart disease. Circulation 1998; 97: 437-43.

21 Bostom AG, Selhub J. Homocysteine and arteriosclerosis. Subclinical and clinical disease associations. Circulation 1999; 99: 2361-3.

22 Corr PB, Saffitz JE, Sobel BE. What is the contribution of altered lipid metabolism to arrhythmogenesis in the ischemic heart?. In: Hearse DJ, Manning AS, Janse MJ, eds. Life Threatening Arrhythmias during Ischemia and Infarction. New York, NY: Raven Press, 1987; 91-114.

23 Parratt JR, Coker SJ, Wainwright CL. Eicosanoids and susceptibility to ventricular arrhythmias during myocardial ischemia and reperfusion. Journal of Molecular and Cellular Cardiology 1987; 19(Suppl. 5): 55-66.

24 Sellmayer A, Witzgall H, Lorenz RL. Effects of dietary fish oil on ventricular premature complexes. American Journal of Cardiology 1995; 76: 974-7.

25 Christensen JH, Gustenhoff P, Korup E. Effect of fish oil on heart rate variability in survivors of myocardial infarction: a double blind randomised controlled trial. British Medical Journal 1996; 312: 677-8.

26 Christensen JH, Christensen MS, Dyerberg J, Schmidt EB. Heart rate variability and fatty acid content of blood cell membranes: a dose-response study with $n-3$ fatty acids. American Journal of Clinical Nutrition 1999; 70: 331-7.

27 Farrell TG, Bashir Y, Cripps T. Risk stratification for arrhythmic events in postinfarction patients based on heart rate variability, ambulatory electrocardiographic variables and the signal-averaged electrocardiogram. Journal of the American College of Cardiology 1991; 18: 687-97.

28 Siscovick DS, Raghunathan TE, King I. Dietary intake and cell membrane levels of long-chain $n-3$ polyunsaturated fatty acids and the risk of primary cardiac arrest. Journal of the American Medical Association 1995; 274: 1363-7.

29 Albert CM, Hennekens CH, O'Donnel CJ. Fish consumption and the risk of sudden cardiac death. Journal of the American Medical Association 1998; 279: 23-8.

30 Leaf A, Albert C, Josephson M, Steinhaus D, Kluger J, Kang JX, et al. Prevention of fatal arrhythmias in high-risk subjects by fish oil $n-3$ fatty acid intake. Circulation 2005; 112: 2762-8.

31 Raitt MH, Connor WE, Morris C, Kron J, Halperin B, Chug SS, et al. Fish oil supplementation and risk of ventricular tachycardia and ventricular fibrillation in patients with implantable defibrillators. Journal of the American Medical Association 2005; 293: 2884-91.

32 Barzi F, Woodward M, Marfisi RM. Mediterranean diet and all-causes mortality after myocardial infarction: results from the GISSI-Prevenzione trial. European Journal of Clinical Nutrition 2003; 57: 604-11.

33 Trichopoulou A, Costacou T, Barnia C, Trichopoulos D. Adherence to a Mediterranean and survival in a Greek population. New England Journal of Medicine 2003; 348: 2599-608.

34 Kris-Etherton P, Eckel R, Howard B, Jeor S St, Bazzarre T. Lyon. Diet Heart Study. Benefits of a Mediterranean-style, National Cholesterol Education Program/American Heart Association Step I Dietary Pattern on cardiovascular disease. Circulation 2001; 103: 1823-5.

35 Chrysohoou C, Panagiotakos D, Pitsavos C. Adherence to the Mediterranean diet attenuates inflammation and coagulation process in healthy adults. The ATTICA Study. Journal of the American College of Cardiology 2004; 44: 152-8.

36 Executive Summary of the Third Report of the National Cholesterol Education Program (NCEP) Expert Panel on the Detection, Evaluation and Treatment of High Blood Cholesterol in Adults (Adult Treatment Panel III). Journal of American Medical Association 2001; 285: 2486-97.

37 Esposito K, Marfella R, Ciotola M, Di Paolo C. Effects of a Mediterranean-style diet on endothelial dysfunction and markers of vascular inflammation in the metabolic syndrome. A randomized trial. Journal of the American Medical Association 2004; 292: 1440-6 